\title{
NON-AUTONOMOUS 2D NAVIER-STOKES SYSTEM WITH A SIMPLE GLOBAL ATTRACTOR AND SOME AVERAGING PROBLEMS*
}

\author{
V.V. ChePyZHov ${ }^{1}$ AND M.I. VISHIK ${ }^{1}$
}

\begin{abstract}
We study the global attractor of the non-autonomous 2D Navier-Stokes system with timedependent external force $g(x, t)$. We assume that $g(x, t)$ is a translation compact function and the corresponding Grashof number is small. Then the global attractor has a simple structure: it is the closure of all the values of the unique bounded complete trajectory of the Navier-Stokes system. In particular, if $g(x, t)$ is a quasiperiodic function with respect to $t$, then the attractor is a continuous image of a torus. Moreover the global attractor attracts all the solutions of the NS system with exponential rate, that is, the attractor is exponential. We also consider the $2 \mathrm{D}$ Navier-Stokes system with rapidly oscillating external force $g(x, t, t / \varepsilon)$, which has the average as $\varepsilon \rightarrow 0+$. We assume that the function $g(x, t, z)$ has a bounded primitive with respect to $z$ and the averaged NS system has a small Grashof number that provides a simple structure of the averaged global attractor. Then we prove that the distance from the global attractor of the original NS system to the attractor of the averaged NS system is less than a small power of $\varepsilon$.
\end{abstract}

Mathematics Subject Classification. 35B40, 35Q30, 34C29.

Received March 3, 2002.

In the first part of the paper we study the global attractor for the 2D Navier-Stokes system with time-dependent external force $g(x, t), t \in \mathbb{R}$. We assume that the function $g(x, t)$ is translation compact in the space $L_{2}^{\text {loc }}(\mathbb{R} ; H)$, that is, the family of translations in time $\{g(\cdot, t+h), h \in \mathbb{R}\}$ forms a precompact set in $L_{2}^{\text {loc }}\left(\left[T_{1}, T_{2}\right] ; H\right)$, where $\left[T_{1}, T_{2}\right]$ is an arbitrary fixed interval of the time axis $\mathbb{R}$. In this case the 2D Navier-Stokes system has the global attractor $\mathcal{A}$. We recall the definition, the construction, and the properties of the global attractor $\mathcal{A}$ in Section 1 and in the beginning of Section 2. Much attention is given to the case, where the Grashof number $G$ of the Navier-Stokes system satisfies the inequality $G<1 / c_{0}$ (see Sect. 2). In this case, we prove that the system has the unique solution $\{z(x, t), t \in \mathbb{R}\}$ bounded in $H$. Then the global attractor $\mathcal{A}$ coincides with the closure in $H$ of the values of the function $z(t): \mathcal{A}=\left[\{z(\cdot, t) \mid t \in \mathbb{R}]_{H}\right.$. Moreover the global attractor $\mathcal{A}$ attracts all the solutions of the system with exponential rate, that is, global attractor $\mathcal{A}$ is exponential. We also prove that if the external force $g(\cdot, t)$ is an almost periodic function in time $t$ with values in $H$, then the corresponding unique bounded solution $z(\cdot, t)$ is almost periodic as well; similarly, if $g(\cdot, t)$ is quasiperiodic, then $z(\cdot, t)$ is quasiperiodic. More exactly, let $g(x, t)=\varphi\left(x, \alpha_{1} t, \ldots, \alpha_{l} t\right)$, where $\varphi\left(x, \omega_{1}, \ldots, \omega_{l}\right)$ is a $2 \pi$-periodic function with respect to each $\omega_{i}, i=1, \ldots, l$ and the numbers $\alpha_{1}, \ldots, \alpha_{l}$ (frequencies) are rationally independent. Then the quasiperiodic

Keywords and phrases: Non-autonomous Navier-Stokes system, global attractor, time averaging.

* The research described in this publication was made possible in part by Award of the US Civilian Research ES Development Foundation for the Independent States of the Former Soviet Union (CRDF) RM1-2343-MO-02, by Grant of INTAS No. 899, and by Grant of Russian Foundation of Basic Researches.

1 Institute for Information Transmission Problems, Russian Academy of Sciences, B. Karetniy 19, Moscow 101447, GSP-4, Russia; e-mail: vishik@iitp.ru 
solution $z(x, t)$ has the same frequencies $\alpha_{1}, \ldots, \alpha_{l}$ and the global attractor $\mathcal{A}$ is a Lipschitz-continuous image of an $l$-dimensional torus $\mathbb{T}^{l}$ into $H$ :

$$
\mathcal{A}=\Phi\left(\mathbb{T}^{l}\right), \Phi: \mathbb{T}^{l} \rightarrow H
$$

In Section 3 we study the Navier-Stokes system with external force of the form $g(x, t, t / \varepsilon)$. Let us formulate the main assumptions for this function. We assume that the function $g(\cdot, t, t / \varepsilon)$ has the uniformly bounded norm in $L_{2}(\tau, \tau+1 ; H)$ and the function $g^{\varepsilon}(t)=g(\cdot, t, t / \varepsilon)$ has the uniform average $g^{0}(t)=\bar{g}(\cdot, t)$ as $\varepsilon \rightarrow 0+$ (see Sect. 3 for more details). The essential assumption is the following: there exists a function $J(x, t, z)$ such that $\partial_{z} J(x, t, z)=g(x, t, z)$ and $\|J(\cdot, t, z)\|_{H} \leq M$ for all $(t, z) \in \mathbb{R} \times \mathbb{R}$. In Section 3 , the sufficient conditions for the existence of such function $J$ are given. We also assume that the Grashof number $G^{0}$ of the averaged 2D Navier-Stokes system with external force $\bar{g}(x, t)$ satisfies the inequality $G^{0}<1 / c_{0}$. Notice that the Grashof numbers $G^{\varepsilon}$ of the systems with external forces $g(x, t, t / \varepsilon)$ can be large. Let $\mathcal{A}^{\varepsilon}$ be the corresponding global attractor of the system with external force $g(x, t, t / \varepsilon)$ and let $\mathcal{A}^{0}$ be the global attractor of the averaged system. We prove the following estimate for the Hausdorff distance from $\mathcal{A}^{\varepsilon}$ to $\mathcal{A}^{0}$ :

$$
\operatorname{dist}_{H}\left(\mathcal{A}^{\varepsilon}, \mathcal{A}^{0}\right) \leq C \varepsilon^{\gamma}, 0<\gamma<1
$$

where the number $\gamma$ has an explicit expression.

In Section 4 we study the case, where the function $g(x, t, z)$ is quasiperiodic in both variables $t$ and $z$. Assume that the conditions of Section 3 hold and $G^{\varepsilon}<1 / c_{0}$. Then both global attractors $\mathcal{A}^{\varepsilon}$ and $\mathcal{A}^{0}$ are images of the corresponding tori and the above estimate for the distance $\operatorname{dist}_{H}\left(\mathcal{A}^{\varepsilon}, \mathcal{A}^{0}\right)$ holds.

Finally we note that in Section 1 the general scheme for the construction of the global attractor of a nonautonomous evolution equation is given. The results proved in the paper can be applied to various nonautonomous evolution equations of mathematical physics that satisfies the similar conditions.

\section{Global attractors of nON-Autonomous evolution EQUations}

1. We consider a non-autonomous evolution equation of the form:

$$
\partial_{t} u=A(u, t), t \geq \tau(\tau \in \mathbb{R})
$$

Here $A(u, t)$ denotes a nonlinear operator $A(\cdot, t): E_{1} \rightarrow E_{0}$ for every $t \in \mathbb{R}$, where $E_{1}$ and $E_{0}$ are Banach spaces such that $E_{1} \subseteq E_{0}$. We study solutions $u(t)$ of this equation that are defined for all $t \geq \tau$. For $t=\tau$ we consider the initial condition:

$$
u(\tau)=\left.u\right|_{t=\tau}=u_{\tau}, u_{\tau} \in E,
$$

where $E$ is a Banach space such that $E_{1} \subseteq E \subseteq E_{0}$. We assume that for every $\tau \in \mathbb{R}$ and for all $u_{\tau} \in E$ Cauchy problem $(1,2)$ has a unique solution $u(t)$ such that $u(t) \in E$ for all $t \geq \tau$. The meaning of the expression "the function $u(t)$ is a solution of problem $(1,2)$ " should be clarified in each particular case. We study the following two-parametric family of operators $\{U(t, \tau)\}, t \geq \tau, \tau \in \mathbb{R}$ generated by problem $(1,2)$ and acting in $E$ by the formula

$$
U(t, \tau) u_{\tau}=u(t), t \geq \tau, \tau \in \mathbb{R}
$$

where $u(t)$ is a solution of $(1,2)$ with initial data $u_{\tau} \in E$. Since the Cauchy problem $(1,2)$ is uniquely solvable, the family of operators $\{U(t, \tau)\}$ satisfies the following properties:

1. $U(\tau, \tau)=I d$ for all $\tau \in \mathbb{R}$, where $I d$ is the identity operator;

2. $U(t, s) \circ U(s, \tau)=U(t, \tau)$ for all $t \geq s \geq \tau, \tau \in \mathbb{R}$. 
The family of operators $\{U(t, \tau)\}$ is called the process generated by problem $(1,2)$.

We now define the notion of the global attractor $\mathcal{A}$ of the process $\{U(t, \tau)\}$ generated by problem $(1,2)$. By $\mathcal{B}(E)$ we denote the family of all bounded sets in $E$. A set $B_{0} \subset E$ is said to be absorbing for the process $\{U(t, \tau)\}$ if for any set $B \in \mathcal{B}(E)$ there is a number $h=h(B)$ such that

$$
U(t, \tau) B \subseteq B_{0} \text { for all } t \text { and } \tau, t-\tau \geq h .
$$

A set $P \subset E$ is said to be attracting for the process $\{U(t, \tau)\}$ if, for every $\varepsilon>0$, the set $\mathcal{O}_{\varepsilon}(P)$ is absorbing for this process (here and below $\mathcal{O}_{\varepsilon}(M)$ denotes an $\varepsilon$-neighborhood of a set $M$ in the space $E$ ), that is, for every bounded set $B \in \mathcal{B}(E)$, there exists a number $h=h(\varepsilon, P)$ such that

$$
U(t, \tau) B \subseteq \mathcal{O}_{\varepsilon}(P) \text { for all } t \text { and } \tau, t-\tau \geq h .
$$

Property (5) can also be formulated in the following manner: for every set $B \in \mathcal{B}(E)$

$$
\sup _{\tau \in \mathbb{R}} \operatorname{dist}_{E}(U(\tau+h, \tau) B, P) \rightarrow 0(h \rightarrow+\infty) .
$$

Here $\operatorname{dist}_{E}(X, Y)$ denotes the Hausdorff distance from the set $X$ to the set $Y$ in the space $E$ :

$$
\operatorname{dist}_{E}(X, Y)=\sup _{x \in X} \inf _{y \in Y}\|x-y\|_{E} .
$$

Definition 1.1. A set $\mathcal{A} \subset E$ is called the global attractor of the process $\{U(t, \tau)\}$ if it is closed in $E$, is attracting for the process $\{U(t, \tau)\}$ and satisfies the following property of minimality: $\mathcal{A}$ belongs to any closed attracting set of the process.

It is easy to see that any process has at most one global attractor. This notion was introduced in [1-3]. A process having a compact absorbing set is called compact and a process having a compact attracting set is called asymptotically compact. The following result holds true:

Theorem 1.1. If a process $\{U(t, \tau)\}$ is uniformly asymptotically compact, then it has the compact in $E$ global attractor.

The proof of this theorem can be found in [3] as well as the following equality for the global attractor $\mathcal{A}$ :

$$
\mathcal{A}=\omega(P):=\bigcap_{h \geq 0}\left[\bigcup_{t-\tau \geq h} U(t, \tau) P\right]_{E},
$$

where $P$ is an arbitrary compact attracting set of the process. In (7) the square brackets $[\cdot]_{E}$ denote the closure in the space $E$.

Remark 1.1. In Theorem 1.1 we do not assume that the process $\{U(t, \tau)\}$ is continuous in $E$. (This assumption was essential in the theorems on the existence of global attractors of semigroups corresponding to autonomous evolution equations.) The reason is that we prove the property of minimality of the global attractor.

2. To describe the general structure of the global attractor of a process we need the notion of the kernel of the process or the kernel of equation (1).

A function $u(s), s \in \mathbb{R}$ with values in $E$ is said to be a complete trajectory of the process $\{U(t, \tau)\}$ if

$$
U(t, \tau) u(\tau)=u(t) \text { for all } t \geq \tau, \tau \in \mathbb{R} .
$$

A complete trajectory $u(s)$ is called bounded if the set $\{u(s), s \in \mathbb{R}\}$ is bounded in $E$. 
Definition 1.2. The kernel $\mathcal{K}$ of the process $\{U(t, \tau)\}$ is the family of all bounded complete trajectories of this process:

$$
\mathcal{K}=\left\{u(\cdot) \mid u \text { satisfies (8) and }\|u(s)\|_{E} \leq C_{u} \forall s \in \mathbb{R}\right\}
$$

The set

$$
\mathcal{K}(t)=\{u(t) \mid u(\cdot) \in \mathcal{K}\} \subset E, t \in \mathbb{R}
$$

is called the kernel section at time $t$.

It is not difficult to prove the following property:

Proposition 1.1. If the process $\{U(t, \tau)\}$ has the global attractor $\mathcal{A}$, then

$$
\bigcup_{t \in \mathbb{R}} \mathcal{K}(t) \subseteq \mathcal{A}
$$

We note that, in the generic case, inclusion (9) is strict, that is, there exist points of the global attractor $\mathcal{A}$ that are not values of bounded complete trajectories of the original equation (1) (see Rem. 2.2). Nevertheless we shall show that such points lie on the complete trajectories of "contiguous" equations. To describe these "contiguous" equations we introduce the notion of time symbol of the equation under the consideration. We assume that all the terms of equation (1) that depend explicitly on time $t$ can be written as a function $\sigma(t), t \in \mathbb{R}$ with values in an appropriate Banach space $\Psi$. We now rewrite equation (1) itself in the form:

$$
\partial_{t} u=A_{\sigma(t)}(u), t \geq \tau(\tau \in \mathbb{R}) .
$$

The function $\sigma(t)$ is said to be the time symbol of the equation. In applications $\sigma(t)$ consists of the coefficients and terms of the equation that depend on time. For example, in the non-autonomous Navier-Stokes system the symbol is the external force depending on time: $\sigma(t)=g(x, t)$.

We assume that the symbol $\sigma(t)$, as a function of time $t$, belongs to an enveloping space

$$
\Xi=\{\xi(t), t \in \mathbb{R} \mid \xi(t) \in \Psi \text { for almost all } t \in \mathbb{R}\},
$$

equipped with a Hausdorff topology. We assume that the translation group $\{T(h), h \in \mathbb{R}\}$ acting by the formula $T(h) \xi(t)=\xi(h+t)$ is continuous in $\Xi$.

The symbol of the original equation (1) is denoted by $\sigma_{0}(t)$. Along with this equation having the symbol $\sigma_{0}(t)$ we consider equations (10) with symbols $\sigma_{h}(t)=\sigma_{0}(t+h)$ for any $h \in \mathbb{R}$. Moreover, we also consider the equations with symbols $\sigma(t)$ that are limits of the sequences of the form $\sigma_{h_{n}}(t)=\sigma_{0}\left(t+h_{n}\right)$ as $n \rightarrow \infty$ in the space $\Xi$. The resulting family of symbols forms the hull $\mathcal{H}\left(\sigma_{0}\right)$ of the original symbol $\sigma_{0}(t)$ in the space $\Xi$.

Definition 1.3. The set

$$
\mathcal{H}\left(\sigma_{0}\right)=[\{\sigma(t+h) \mid h \in \mathbb{R}\}]_{\Xi}
$$

is called the hull $\mathcal{H}(\sigma)$ of the function $\sigma(t)$ in the space $\Xi$, where $[\cdot]_{\Xi}$ denotes the closure in the topological space $\Xi$.

We are going to study equations of the form (1) and (10), whose symbols $\sigma(t)$ are translation compact functions in $\Xi$.

Definition 1.4. A function $\sigma(t) \in \Xi$ is called translation compact (tr.c.) in $\Xi$, if the hull $H(\sigma)$ is compact in $\Xi$. 
Consider the main examples of translation compact functions that we shall use in this paper.

Example 1.1. Let $\Xi=L_{p}^{\text {loc }}(\mathbb{R} ; \mathcal{E})$, where $p \geq 1$ and $\mathcal{E}$ is a Banach space. The space $L_{p}^{\text {loc }}(\mathbb{R} ; \mathcal{E})$ consists of functions $\xi(t), t \in \mathbb{R}$ with values in $\mathcal{E}$ that are $p$-power locally integrable in the Bochner sense, that is,

$$
\int_{t_{1}}^{t_{2}}\|\xi(t)\|_{\mathcal{E}}^{p} \mathrm{~d} t<+\infty \forall\left[t_{1}, t_{2}\right] \subset \mathbb{R}
$$

We consider the following convergence topology in the space $L_{p}^{\text {loc }}(\mathbb{R} ; \mathcal{E})$. By the definition, $\xi_{n}(t) \rightarrow \xi(t)(n \rightarrow \infty)$ in $L_{p}^{\operatorname{loc}}(\mathbb{R} ; \mathcal{E})$ if

$$
\int_{t_{1}}^{t_{2}}\left\|\xi_{n}(t)-\xi(t)\right\|_{\mathcal{E}}^{p} \mathrm{~d} t \rightarrow 0(n \rightarrow \infty)
$$

for every interval $\left[t_{1}, t_{2}\right] \subset \mathbb{R}$. The space $L_{p}^{\text {loc }}(\mathbb{R} ; \mathcal{E})$ is countably normable, metrizable, and complete. Consider tr.c. functions in the space $L_{p}^{\text {loc }}(\mathbb{R} ; \mathcal{E})$. We have the following criterion (see, for example [3]): a function $\sigma_{0}(t)$ is tr.c. in $L_{p}^{\text {loc }}(\mathbb{R} ; \mathcal{E})$ if and only if (i) for any $h \geq 0$ the set $\left\{\int_{t}^{t+h} \sigma_{0}(s) \mathrm{d} s \mid t \in \mathbb{R}\right\}$ is precompact in $\mathcal{E}$ and (ii) there exists a positive function $\beta(s) \rightarrow 0(s \rightarrow 0+)$ such that

$$
\int_{t}^{t+1}\left\|\sigma_{0}(s)-\sigma_{0}(s+l)\right\|_{\mathcal{E}}^{p} \mathrm{~d} s \leq \beta(|l|) \forall t \in \mathbb{R}
$$

Notice that

$$
\sup _{t \in \mathbb{R}} \int_{t}^{t+1}\left\|\sigma_{0}(s)\right\|_{\mathcal{E}}^{p} \mathrm{~d} s<+\infty \forall t \in \mathbb{R}
$$

for any tr.c. function in $L_{p}^{\text {loc }}(\mathbb{R} ; \mathcal{E})$.

Example 1.2. Similarly we define translation compact functions in the space $C(\mathbb{R} ; \mathcal{E})$ that consists of continuous functions $\xi(t), t \in \mathbb{R}$ with values in $\mathcal{E}$. The space $C(\mathbb{R} ; \mathcal{E})$ is equipped with the uniform convergence topology on every interval $\left[t_{1}, t_{2}\right] \subset \mathbb{R}$ (see $[3]$ ).

Example 1.3. Almost periodic functions with values in $\mathcal{E}$ are tr.c. functions in the space $C(\mathbb{R} ; \mathcal{E})$. Inside the class of a.p. functions we consider a subclass of quasiperiodic functions. A function $\sigma_{0}(t) \in C(R ; E)$ is said to be quasiperiodic (q.p.) if it has the form:

$$
\sigma_{0}(t)=\phi\left(\alpha_{1} t, \alpha_{2} t, \ldots, \alpha_{k} t\right)=\phi(\bar{\alpha} t)
$$

where the function $\phi(\bar{\omega})=\phi\left(\omega_{1}, \omega_{2}, \ldots, \omega_{k}\right)$ is continuous and $2 \pi$-periodic with respect to each argument $\omega_{i} \in \mathbb{R}:$

$$
\phi\left(\omega_{1}, \ldots, \omega_{i}+2 \pi, \ldots, \omega_{k}\right)=\phi\left(\omega_{1}, \ldots, \omega_{i}, \ldots, \omega_{k}\right), i=1, \ldots, k .
$$

We denote by $\mathbb{T}^{k}=[\mathbb{R} \bmod 2 \pi]^{k}$ the $k$-dimensional torus. Then $\phi \in C\left(\mathbb{T}^{k} ; \mathcal{E}\right)$. We assume that the real numbers $\alpha_{1}, \alpha_{2}, \ldots, \alpha_{k}$ in (13) are rationally independent (otherwise we can reduce the number of independent arguments $\omega_{i}$ in the representation (13)). It follows easily that the hull of the q.p. function $\sigma_{0}(t)$ in $C(\mathbb{R} ; \mathcal{E})$ is the following set:

$$
\left\{\varphi\left(\bar{\alpha} t+\bar{\omega}_{1}\right) \mid \bar{\omega}_{1} \in \mathbb{T}^{k}\right\}=\mathcal{H}\left(\sigma_{0}\right), \bar{\alpha}=\left(\alpha_{1}, \alpha_{2}, \ldots, \alpha_{k}\right)
$$


Consequently the set $\mathcal{H}\left(\sigma_{0}\right)$ is a continuous image of the $k$-dimensional torus $\mathbb{T}^{k}$. For $k=1$ we obtain a periodic function.

In [3] other examples of tr.c. functions in $C(\mathbb{R} ; \mathcal{E})$ are given which are not a.p. functions.

3. We now consider a family of equations (10) with symbols $\sigma(t)$ from the hull $\mathcal{H}\left(\sigma_{0}\right)$ of the symbol $\sigma_{0}(t)$ of the original equation. We assume that the function $\sigma_{0}(t)$ is tr.c. in the topological space $\Xi$. For simplicity we assume that the set $\mathcal{H}\left(\sigma_{0}\right)$ is a complete metric space. In all examples given above this assumption holds. We suppose that for every symbol $\sigma \in \mathcal{H}\left(\sigma_{0}\right)$ the Cauchy problem $(10,2)$ has a unique solution for any $\tau \in \mathbb{R}$ and for every initial condition $u_{\tau} \in E$. Thus, we have the family of processes $\left\{U_{\sigma}(t, \tau)\right\}, \sigma \in \mathcal{H}\left(\sigma_{0}\right)$ acting in the space $E$. We note that the following translation identity holds for this family of processes:

$$
U_{T(h) \sigma}(t, \tau)=U_{\sigma}(t+h, \tau+h) \forall h \geq 0, t \geq \tau, \tau \in \mathbb{R} .
$$

Here $T(h) \sigma(t)=\sigma(t+h)$.

We now consider the extended phase space $E \times \mathcal{H}\left(\sigma_{0}\right)$. Using the identity (15) we construct the semigroup $\{S(h), h \geq 0\}$ acting in the space $E \times \mathcal{H}\left(\sigma_{0}\right)$ by the formula:

$$
S(h)(u, \sigma)=\left(U_{\sigma}(h, 0) u, T(h) \sigma\right), h \geq 0 .
$$

The family of processes $\left\{U_{\sigma}(t, \tau)\right\}, \sigma \in \mathcal{H}\left(\sigma_{0}\right)$ is called $\left(E \times \mathcal{H}\left(\sigma_{0}\right), E\right)$-continuous if for any $t$ and $\tau, t \geq \tau$ the mapping $(u, \sigma) \mapsto U_{\sigma}(t, \tau) u$ is continuous from $E \times \mathcal{H}\left(\sigma_{0}\right)$ into $E$.

We denote by $\Pi_{1}$ and $\Pi_{2}$ the projectors acting from $E \times \mathcal{H}\left(\sigma_{0}\right)$ onto $E$ and $\mathcal{H}\left(\sigma_{0}\right)$ by the formulae:

$$
\Pi_{1}(u, \sigma)=u, \Pi_{2}(u, \sigma)=\sigma .
$$

We now formulate the main theorem on the structure of the global attractor of equation (1) with tr.c. symbol $\sigma_{0}(t)$. The corresponding process we denote by $\left\{U_{\sigma_{0}}(t, \tau)\right\}$.

Theorem 1.2. We assume that the function $\sigma_{0}(t)$ is translation compact in $\Xi$. Let the process $\left\{U_{\sigma_{0}}(t, \tau)\right\}$ be asymptotically compact and let the corresponding family of processes $\left\{U_{\sigma}(t, \tau)\right\}, \sigma \in \mathcal{H}\left(\sigma_{0}\right)$ be $\left(E \times \mathcal{H}\left(\sigma_{0}\right), E\right)$ continuous. Then the semigroup $\{S(h)\}$ acting in $E \times \mathcal{H}\left(\sigma_{0}\right)$ by formula (16) has the global attractor $\mathbf{A}, S(h) \mathbf{A}$ $=\mathbf{A}$ for all $h \geq 0$. Moreover

1. $\Pi_{2} \mathbf{A}=\mathcal{H}\left(\sigma_{0}\right)$;

2. $\Pi_{1} \mathbf{A}=\mathcal{A}$ is the global attractor of the process $\left\{U_{\sigma_{0}}(t, \tau)\right\}$;

3. the global attractor $\mathcal{A}$ has the following representation:

$$
\mathcal{A}=\bigcup_{\sigma \in \mathcal{H}\left(\sigma_{0}\right)} \mathcal{K}_{\sigma}(0)=\bigcup_{\sigma \in \mathcal{H}\left(\sigma_{0}\right)} \mathcal{K}_{\sigma}(t)
$$

where $\mathcal{K}_{\sigma}$ is the kernel of the process $\left\{U_{\sigma}(t, \tau)\right\}$ with symbol $\sigma \in \mathcal{H}\left(\sigma_{0}\right)$. Here $t$ is any fixed number. The kernel $\mathcal{K}_{\sigma}$ is non-empty for every $\sigma \in \mathcal{H}\left(\sigma_{0}\right)$.

The detailed proof of Theorem 1.2 can be found in $[2,3]$. For the brevity we study in the next sections the global attractor for the 2D Navier-Stokes system. Notice that the similar results can be established for the non-autonomous dissipative wave equation and for other equations of mathematical physics.

\section{2D NAVIER-Stokes System With translation COMPACT EXTERnAL FORCE}

Excluding the pressure the 2D Navier-Stokes system can be written in the form:

$$
\begin{aligned}
\partial_{t} u & =-\nu L u-B(u, u)+g_{0}(x, t),(\nabla, u)=0, \\
\left.u\right|_{\partial \Omega} & =0, x=\left(x_{1}, x_{2}\right) \in \Omega \Subset \mathbb{R}^{2} .
\end{aligned}
$$


Here $u=u(x, t)=\left(u^{1}(x, t), u^{2}(x, t)\right), L u=-\Pi \Delta u$ is the Stokes operator,

$$
B(u, u)=\Pi\left(u^{1} \partial_{x_{1}} u+u^{2} \partial_{x_{2}} u\right)
$$

$\Pi$ is the orthogonal projector from the space $\left(L_{2}(\Omega)\right)^{2}$ onto the space $H=\left[\mathcal{V}_{0}\right]_{\left(L_{2}(\Omega)\right)^{2}}$, where $\mathcal{V}_{0}:=\left\{v \in\left(C_{0}^{\infty}(\Omega)\right)^{2} \mid(\nabla, v)=0\right\}$. We also denote the space $H_{1} \equiv V:=\left[\mathcal{V}_{0}\right]_{\left(H_{0}^{1}(\Omega)\right)^{2}}$. The norms in the spaces $H$ and $V$ are $|\cdot|$ and $\|\cdot\|$, respectively.

We assume that the external force $g_{0}(\cdot, t) \in H$ for almost every $t \in \mathbb{R}$ and has a finite norm in the space $L_{2}^{b}(\mathbb{R} ; H)$, that is,

$$
\left\|g_{0}\right\|_{L_{2}^{b}(\mathbb{R} ; H)}^{2} \equiv\left\|g_{0}\right\|_{L_{2}^{b}}^{2}:=\sup _{t \in \mathbb{R}} \int_{t}^{t+1}\left|g_{0}(\cdot, s)\right|^{2} \mathrm{~d} s \leq M<+\infty .
$$

We also assume that the function $g_{0}(\cdot, t) \equiv g_{0}(t)$ is translation compact in the space $L_{2}^{\text {loc }}(\mathbb{R} ; H)$. The corresponding necessary and sufficient conditions are given in Section 1. Another sufficient condition is as follows: a function $g_{0}(t)$ is translation compact in $L_{2}^{\text {loc }}(\mathbb{R} ; H)$, if $g_{0} \in L_{2}^{b}\left(\mathbb{R} ; H_{1}\right)$ and $\partial_{t} g_{0} \in L_{2}^{b}\left(\mathbb{R} ; H_{-1}\right)$, where $H_{-1}=\left(H_{1}\right)^{*}$, that is,

$$
\begin{gathered}
\left\|g_{0}\right\|_{L_{2}^{b}\left(\mathbb{R} ; H_{1}\right)}^{2}:=\sup _{t \in \mathbb{R}} \int_{t}^{t+1}\left\|g_{0}(\cdot, s)\right\|^{2} \mathrm{~d} s \leq M_{1}<+\infty \\
\left\|\partial_{t} g_{0}\right\|_{L_{2}^{b}\left(\mathbb{R} ; H_{-1}\right)}^{2}:=\sup _{t \in \mathbb{R}} \int_{t}^{t+1}\left\|\partial_{t} g_{0}(\cdot, s)\right\|_{H_{-1}}^{2} \mathrm{~d} s \leq M_{-1}<+\infty
\end{gathered}
$$

(see $[3])$. We denote by $\mathcal{H}\left(g_{0}\right)$ the hull of the function $g_{0}$ in the space $L_{2}^{\text {loc }}(\mathbb{R} ; H)$. It is clear that

$$
\|g\|_{L_{2}^{b}}^{2} \leq\left\|g_{0}\right\|_{L_{2}^{b}}^{2} \leq M
$$

for every function $g \in \mathcal{H}\left(g_{0}\right)$.

Consider the following initial conditions for equations (18):

$$
\left.u\right|_{t=\tau}=u_{\tau}, u_{\tau} \in H(\tau \in \mathbb{R})
$$

Recall that the Cauchy problem $(18,22)$ has a unique solution $u(t) \in C\left(\mathbb{R}_{\tau} ; H\right) \cap L_{2}^{b}\left(\mathbb{R}_{\tau} ; V\right)$ such that $\partial_{t} u$ $\in L_{2}^{b}\left(\mathbb{R}_{\tau} ; H_{-1}\right), \mathbb{R}_{\tau}=[\tau,+\infty)$ (see [3-7]). A solution $u(t)$ from this space satisfies equation (18) in the distribution sense of the space $\mathcal{D}^{\prime}\left(\mathbb{R}_{\tau} ; H_{-1}\right)$. Consequently, problem $(18,22)$ generate the process $\left\{U_{g_{0}}(t, \tau)\right\}$ acting in $H$ by the formula $U_{g_{0}}(t, \tau) u_{\tau}=u(t)$, where $u(t)$ is a solution of $(18,22)$. Recall that the process $\left\{U_{g_{0}}(t, \tau)\right\}$ has the absorbing set $B_{0}$ :

$$
B_{0}=\left\{u \in H|| u \mid \leq R_{0}\right\}, R_{0}^{2}=(\nu \lambda)^{-1}\left(1+(\nu \lambda)^{-1}\right)\left\|g_{0}\right\|_{L_{2}^{b}}^{2}
$$

where $\lambda$ is the first eigenvalue of the Stokes operator $L$. Besides the set

$$
B_{1}=\bigcup_{\tau \in \mathbb{R}} U_{g_{0}}(\tau+1, \tau) B_{0}
$$

is also absorbing. Moreover, $B_{1}$ is bounded in $V=H_{1}$ and, therefore, compact in $H$ (see [3]). Thus, the process $\left\{U_{g_{0}}(t, \tau)\right\}$ is compact in $H$. Applying Theorem 1.1 from Section 1 we conclude that the process $\left\{U_{g_{0}}(t, \tau)\right\}$ has 
the global attractor $\mathcal{A}$ and the following equality holds:

$$
\mathcal{A}=\bigcap_{h \geq 0}\left[\bigcup_{t-\tau \geq h} U_{g_{0}}(t, \tau) B_{0}\right]_{H} .
$$

The set $\mathcal{A}$ is bounded in $H_{1}$.

The symbol of equation (18) is the function $g_{0}(t)=\sigma_{0}(t)$. We note that, for every symbol $g \in \mathcal{H}\left(g_{0}\right)$, the corresponding problem $(18,22)$ is uniquely solvable. Hence, the family of processes $\left\{U_{g}(t, \tau)\right\}, g \in \mathcal{H}\left(g_{0}\right)$ acting $H$ is defined. In [3] it is proved that this family is $\left(H \times \mathcal{H}\left(g_{0}\right)\right)$-continuous. Therefore Theorem 1.2 implies the equality

$$
\mathcal{A}=\bigcup_{g \in \mathcal{H}\left(g_{0}\right)} \mathcal{K}_{g}(0)
$$

where $\mathcal{K}_{g}$ is the kernel of the process $\left\{U_{g}(t, \tau)\right\}$, which consists of all the bounded complete solutions $u_{g}(t), t \in \mathbb{R}$ of the Navier-Stokes system with external force $g(t)$. The kernel $\mathcal{K}_{g}$ is non-empty for every $g \in \mathcal{H}\left(g_{0}\right)$. Notice that

$$
\begin{aligned}
& \mathcal{A} \subset B_{0}=B_{R_{0}}(0), R_{0}^{2}=(\nu \lambda)^{-1}\left(1+(\nu \lambda)^{-1}\right)\left\|g_{0}\right\|_{L_{2}^{b}}^{2}, \\
& \mathcal{A} \subset B_{1}, B_{1}=\left\{u \in V \mid\|v\| \leq R^{\prime}\right\},
\end{aligned}
$$

where $R^{\prime}$ depends on $\nu, \lambda$, and $\left\|g_{0}\right\|_{L_{2}^{b}}^{2}$. In particular we conclude from (25) that

$$
\left\|u_{g}(t)\right\| \leq R^{\prime} \forall t \in \mathbb{R}
$$

for every function $u_{g}(\cdot) \in \mathcal{K}_{g}, g \in \mathcal{H}\left(g_{0}\right)$. We also need the well-known energy inequality:

$$
|u(t)|^{2}+\nu \int_{\tau}^{t}\|u(s)\|^{2} \mathrm{~d} s \leq|u(\tau)|^{2}+(\nu \lambda)^{-1} \int_{\tau}^{t}|g(s)|^{2} \mathrm{~d} s,
$$

where $u(t)=U_{g}(t, \tau) u(\tau)($ see $[3-7])$.

We now consider the important particular case of system (18). Let the Grashof number $G$ of the NavierStokes system satisfies the following inequality:

$$
G:=\frac{\left\|g_{0}\right\|_{L_{2}^{b}}}{\lambda \nu^{2}}<\frac{1}{c_{0}}
$$

where the constant $c_{0}$ is taken from the inequality

$$
|(B(v, w), v)| \leq c_{0}|v|\|v\|\||| w\|
$$

which holds for all $v, w \in V$.

Then the Navier-Stokes system

$$
\partial_{t} u=-\nu L u-B(u, u)+g(t)
$$

has the unique solution $z_{g}(t), t \in \mathbb{R}$ bounded in $H$ for every $g \in \mathcal{H}\left(g_{0}\right)$, that is, the kernel $\mathcal{K}_{g}$ consists of the unique trajectory $z_{g}(t)$. This solution $z_{g}(t)$ is exponentially stable, i.e., for every solution $u_{g}(t)$ of equation (32) 
the following inequality holds:

$$
\left|u_{g}(t)-z_{g}(t)\right| \leq C_{0}\left|u_{\tau}-z_{g}(\tau)\right| \mathrm{e}^{-\beta(t-\tau)} \forall t \geq \tau,
$$

where $u_{g}(t)=U_{g}(t, \tau) u_{\tau}$ (the constants $C_{0}$ and $\beta$ are independent of $u_{\tau}$ and $\tau$ ).

Let us prove the above assertion and inequality (33). By (25), at least one bounded solution $z_{g}(t):=z(t)$ exists. Let $u_{g}(t):=u(t)$ be an arbitrary solution of $(32)$. The function $w(t)=u(t)-z(t)$ satisfies the equation

$$
\partial_{t} w+\nu L w+B(w, w+z)+B(z, w)=0 .
$$

Multiplying by $w$ and using the well-known identity $(B(z, w), w)=0$ and (31) we obtain that

$$
\partial_{t}|w|^{2}+2 \nu\|w\|^{2}=2(B(w, z), w) \leq 2 c_{0}|w|\|w\|\|z\| \leq \nu\|w\|^{2}+c_{0}^{2} \nu^{-1}|w|^{2}\|z\|^{2} .
$$

Since $\lambda|w|^{2} \leq\|w\|^{2}$, we have

$$
\partial_{t}|w|^{2}+\nu \lambda|w|^{2} \leq \partial_{t}|w|^{2}+\nu\|w\|^{2} \leq c_{0}^{2} \nu^{-1}|w|^{2}\|z\|^{2} .
$$

Consequently,

$$
\partial_{t}|w|^{2}+\left(\nu \lambda-c_{0}^{2} \nu^{-1}\|z(t)\|^{2}\right)|w|^{2} \leq 0 .
$$

Multiplying this inequality by $\exp \left\{\int_{\tau}^{t}\left(\nu \lambda-c_{0}^{2} \nu^{-1}\|z(s)\|^{2}\right) \mathrm{d} s\right\}$ and integrating over $[\tau, t]$, we obtain

$$
\begin{aligned}
|w(t)|^{2} & \leq|w(\tau)|^{2} \exp \left\{\int_{\tau}^{t}\left(-\nu \lambda+c_{0}^{2} \nu^{-1}\|z(s)\|^{2}\right) \mathrm{d} s\right\} \\
& =|w(\tau)|^{2} \exp \left\{-\nu \lambda(t-\tau)+c_{0}^{2} \nu^{-1} \int_{\tau}^{t}\|z(s)\|^{2} \mathrm{~d} s\right\}
\end{aligned}
$$

By (29) we find that

$$
\begin{aligned}
\int_{\tau}^{t}\|z(s)\|^{2} \mathrm{~d} s & \leq \nu^{-1}|z(\tau)|^{2}+\left(\nu^{2} \lambda\right)^{-1} \int_{\tau}^{t}|g(s)|^{2} \mathrm{~d} s \\
& \leq \nu^{-1}|z(\tau)|^{2}+\left(\nu^{2} \lambda\right)^{-1}(t-\tau+1)\|g\|_{L_{2}^{b}}^{2} \\
& \leq \nu^{-1}|z(\tau)|^{2}+\left(\nu^{2} \lambda\right)^{-1}(t-\tau+1)\left\|g_{0}\right\|_{L_{2}^{b}}^{2} .
\end{aligned}
$$

Since $z(\tau) \in \mathcal{A}_{\mathcal{H}\left(g_{0}\right)}$, it follows from (26) that

$$
|z(\tau)|^{2} \leq(\nu \lambda)^{-1}\left(1+(\nu \lambda)^{-1}\right)\left\|g_{0}\right\|_{L_{2}^{b}}^{2}=R_{0}^{2} .
$$

Hence,

$$
\begin{aligned}
\int_{\tau}^{t}\|z(s)\|^{2} \mathrm{~d} s & \leq\left(\nu^{-1} R_{0}^{2}+\left(\nu^{2} \lambda\right)^{-1}\left\|g_{0}\right\|_{L_{2}^{b}}^{2}\right)+\left(\nu^{2} \lambda\right)^{-1}(t-\tau)\left\|g_{0}\right\|_{L_{2}^{b}}^{2} \\
& =R_{1}^{2}+\left(\nu^{2} \lambda\right)^{-1}(t-\tau)\left\|g_{0}\right\|_{L_{2}^{b}}^{2},
\end{aligned}
$$

where $R_{1}^{2}=\nu^{-1} R_{0}^{2}+\left(\nu^{2} \lambda\right)^{-1}\left\|g_{0}\right\|_{L_{2}^{b}}^{2}$. Substituting this estimate into (35) we obtain the inequality

$$
|w(t)|^{2} \leq|w(\tau)|^{2} C_{0} \exp (-\beta(t-\tau)),
$$


where

$$
\beta=\nu \lambda-c_{0}^{2}\left(\nu^{3} \lambda\right)^{-1}\left\|g_{0}\right\|_{L_{2}^{b}}^{2} \text { and } C_{0}=\exp \left(c_{0}^{2} \nu^{-1} R_{1}^{2}\right)
$$

Notice that

$$
\nu^{-4} \lambda^{-2}\left\|g_{0}\right\|_{L_{2}^{b}}^{2}=G^{2}<\frac{1}{c_{0}^{2}}
$$

and therefore $\beta=\nu \lambda c_{0}^{4}\left(c_{0}^{-2}-\nu^{-4} \lambda^{-2}\left\|g_{0}\right\|_{L_{2}^{b}}^{2}\right)>0$. This implies that

$$
|w(t)|^{2}=|u(t)-z(t)|^{2} \leq|u(\tau)-z(\tau)|^{2} C_{0} \mathrm{e}^{-\beta(t-\tau)} .
$$

Let us show that such a $z(t)$ is unique. If there are two bounded complete solutions $z_{1}(t)$ and $z_{2}(t), t \in \mathbb{R}$, then by $(33)$

$$
\left|z_{1}(t)-z_{2}(t)\right|^{2} \leq\left|z_{1}(\tau)-z_{2}(\tau)\right|^{2} C_{0} \mathrm{e}^{-\beta(t-\tau)} \leq C_{1} C_{0} \mathrm{e}^{-\beta(t-\tau)} .
$$

Letting $\tau \rightarrow-\infty$ we obtain $\left|z_{1}(t)-z_{2}(t)\right|^{2}=0$ for all $t \in \mathbb{R}$.

Properties (33) and (25) implies that the set

$$
\mathcal{A}=\left[\left\{z_{g_{0}}(t) \mid t \in \mathbb{R}\right\}\right]_{H}=\bigcup_{g \in \mathcal{H}\left(g_{0}\right)}\left\{z_{g}(0)\right\}
$$

is the global attractor of the original equation (18) under condition (30).

Remark 2.1. In the work [8] it is shown that $c_{0}=1 / \sqrt{2 \pi}$. It is possible that this constant is the best for inequality (31).

Remark 2.2. It is easy to construct examples of functions $g_{0}(x, t)$ satisfying (30) such that the $\operatorname{set}\left\{z_{g_{0}}(t) \mid t \in \mathbb{R}\right\}$ is not closed in $H$. Nevertheless, the set $\mathcal{A}$ is always closed and to describe this set we need to consider all the functions $z_{g}(t)$ from the kernels of equations with external forces $g \in \mathcal{H}\left(g_{0}\right)$.

Remark 2.3. Inequality (33) implies that the global attractor $\mathcal{A}$ of system (18) is exponential under the condition (30).

We now formulate some corollaries for some special cases of functions $g \in \mathcal{H}\left(g_{0}\right)$.

Corollary 2.1. Let a function $g(t)$ be periodic with period $p$. Then the function $z_{g}(t)$ has the period $p$ as well.

Proof. Consider the corresponding bounded complete trajectory $z_{g}(t)$. Consider also the function $z_{g}(t+p)$ that is, obviously, also a bounded complete trajectory of equation (18) with external force $g(t+p) \equiv g(t)$. Therefore, this function belongs to the kernel $\mathcal{K}_{g}$, which consists of the unique trajectory $z_{g}(t)$. Hence, $z_{g}(t+p) \equiv z_{g}(t)$.

Corollary 2.2. If a function $g(t) \in \mathcal{H}\left(g_{0}\right)$ is almost periodic, then the function $z_{g}(t)$ is almost periodic as well.

Proof. Consider the function $w(t)=z(t)-z(t+p)$, where $z(t):=z_{g}(t)$ and $p$ is an arbitrary fixed number. Similarly to (34) we obtain the following inequality:

$$
\partial_{t}|w|^{2}+\left(\nu \lambda-c_{0}^{2} \nu^{-1}\|z(t)\|^{2}\right)|w|^{2} \leq 2|w| \cdot|g(t)-g(t+p)|,
$$

which implies that

$$
\partial_{t}|w|^{2}+\left(\nu \lambda-c_{0}^{2} \nu^{-1}\|z(t)\|^{2}-\delta\right)|w|^{2} \leq \delta^{-1}|g(t)-g(t+p)|^{2}
$$


Here $\delta$ is a fixed positive number specified below. We also get from the energy inequality (29) that

$$
\begin{aligned}
\nu \int_{\tau}^{t}\|z(s)\|^{2} \mathrm{~d} s & \leq|z(\tau)|^{2}+(\nu \lambda)^{-1} \int_{\tau}^{t}|g(s)|^{2} \mathrm{~d} s \leq|z(\tau)|^{2}+(\nu \lambda)^{-1}(t-\tau+1)\|g\|_{L_{2}^{b}}^{2} \\
& \leq|z(\tau)|^{2}+(\nu \lambda)^{-1}(t-\tau+1)\left\|g_{0}\right\|_{L_{2}^{b}}^{2} .
\end{aligned}
$$

Since $z(\tau) \in \mathcal{A}$, from $(26)$ we have

$$
|z(\tau)|^{2} \leq(\nu \lambda)^{-1}\left(1+(\nu \lambda)^{-1}\right)\left\|g_{0}\right\|_{L_{2}^{b}}^{2}=R_{0}^{2} .
$$

Consequently due to (38) we obtain:

$$
\begin{aligned}
\int_{\tau}^{t}\|z(s)\|^{2} \mathrm{~d} s & \leq\left(\nu^{-1} R_{0}^{2}+\left(\nu^{2} \lambda\right)^{-1}\left\|g_{0}\right\|_{L_{2}^{b}}^{2}\right)+\left(\nu^{2} \lambda\right)^{-1}(t-\tau)\left\|g_{0}\right\|_{L_{2}^{b}}^{2} \\
& =R_{1}^{2}+\left(\nu^{2} \lambda\right)^{-1}(t-\tau)\left\|g_{0}\right\|_{L_{2}^{b}}^{2}
\end{aligned}
$$

where $R_{1}^{2}=\nu^{-1} R_{0}^{2}+\left(\nu^{2} \lambda\right)^{-1}\left\|g_{0}\right\|_{L_{2}^{b}}^{2}$.

We denote $\alpha(t)={ }^{2} \nu \lambda-c_{0}^{2} \nu^{-1}\|z(t)\|^{2}-\delta$. Multiplying equation $\exp \left\{\int_{\tau}^{t} \alpha(s) \mathrm{d} s\right\}$ and integrating over $[\tau, t]$ we find that

$$
|w(t)|^{2} \leq|w(\tau)|^{2} \mathrm{e}^{-\int_{\tau}^{t} \alpha(s) \mathrm{ds}}+\frac{1}{\delta} \int_{\tau}^{t}|g(\theta)-g(\theta+p)|^{2} \mathrm{e}^{-\int_{\theta}^{t} \alpha(s) \mathrm{d} s} \mathrm{~d} \theta
$$

Using (39) we have

$$
\begin{aligned}
-\int_{\theta}^{t} \alpha(s) \mathrm{d} s & \leq c_{0}^{2} \nu^{-3} \lambda^{-1}\left\|g_{0}\right\|_{L_{2}^{b}}^{2}(t-\theta)-(\nu \lambda-\delta)(t-\theta)+c_{0}^{2} \nu^{-1} R_{1}^{2} \\
& =-\left(\nu \lambda-c_{0}^{2} \nu^{-3} \lambda^{-1}\left\|g_{0}\right\|_{L_{2}^{b}}^{2}-\delta\right)(t-\theta)+R_{2}^{2} \\
& =-(\beta-\delta)(t-\theta)+R_{2}^{2},
\end{aligned}
$$

where $R_{2}^{2}=c_{0}^{2} \nu^{-1} R_{1}^{2}$ and $\beta=\nu \lambda-c_{0}^{2} \nu^{-3} \lambda^{-1}\left\|g_{0}\right\|_{L_{2}^{b}}^{2}$. We note that $\nu^{-4} \lambda^{-2}\left\|g_{0}\right\|_{L_{2}^{b}}^{2}=G^{2}<c_{0}^{-2}$ (see (30)) and therefore $\beta=\nu \lambda-c_{0}^{2} \nu^{-3} \lambda^{-1}\left\|g_{0}\right\|_{L_{2}^{b}}^{2}>0$. We now set $\delta=\beta / 2$. Then (40) implies that

$$
|w(t)|^{2} \leq|w(\tau)|^{2} \mathrm{e}^{R_{2}^{2}} \mathrm{e}^{-\beta(t-\tau) / 2}+\frac{2}{\beta} \mathrm{e}^{R_{2}^{2}} \int_{\tau}^{t}|g(\theta)-g(\theta+p)|^{2} \mathrm{e}^{-\beta(t-\theta) / 2} \mathrm{~d} \theta
$$

Let the number $p$ be an $\varepsilon$-period of the function $g$, i.e. $|g(\theta)-g(\theta+p)| \leq \varepsilon$ for all $\theta \in \mathbb{R}$. Then by (42) we have

$$
\begin{aligned}
|w(t)|^{2} & \leq|w(\tau)|^{2} C_{2} \mathrm{e}^{-\beta(t-\tau) / 2}+C_{2} \frac{2}{\beta} \varepsilon^{2} \int_{\tau}^{t} \mathrm{e}^{-\beta(t-\theta) / 2} \mathrm{~d} \theta \\
& \leq|w(\tau)|^{2} C_{2} \mathrm{e}^{-\beta(t-\tau) / 2}+C_{2}\left(\frac{2 \varepsilon}{\beta}\right)^{2}\left(1-\mathrm{e}^{-\beta(t-\tau) / 2}\right) \\
& \leq|w(\tau)|^{2} C_{2} \mathrm{e}^{-\beta(t-\tau) / 2}+C_{2}\left(\frac{2 \varepsilon}{\beta}\right)^{2}, \text { where } C_{2}=\mathrm{e}^{R_{2}^{2}} .
\end{aligned}
$$


Notice that $|w(\tau)| \leq C^{\prime}$ for all $\tau \in \mathbb{R}$. Therefore using (43) and letting $\tau \rightarrow-\infty$ we obtain the inequality

$$
|w(t)|=|z(t)-z(t+p)| \leq \varepsilon \frac{2 \sqrt{C_{2}}}{\beta} .
$$

Hence, $p$ is also $\varepsilon \frac{2 \sqrt{C_{2}}}{\beta}$-period of the function $z(t)$. Then it is straightforward that the function $z(t)$ is almost periodic.

We now study the case, where the function $g_{0}(t)$ is quasiperiodic, that is,

$$
g_{0}(x, t)=\phi\left(x, \alpha_{1} t, \ldots, \alpha_{k} t\right)=\phi(x, \bar{\alpha} t),
$$

$\phi(\cdot, \bar{\omega}) \in C^{\mathrm{Lip}}\left(\mathbb{T}^{k} ; H\right), \bar{\omega}=\left(\omega_{1}, \ldots, \omega_{k}\right)$, and real numbers $\left(\alpha_{1}, \ldots, \alpha_{k}\right)=\bar{\alpha}$ are rationally independent.

Theorem 2.1. Let the condition (30) hold and let the function $g_{0}(t)$ be quasiperiodic. Then the function $z_{0}(t)=z_{g_{0}}(t)$ is also quasiperiodic, that is, there exists a function $\Phi(x, \bar{\omega}) \in C^{\operatorname{Lip}}\left(\mathbb{T}^{k} ; H\right)$ such that $z_{0}(x, t)$ $=\Phi\left(x, \alpha_{1} t, \ldots, \alpha_{k} t\right)$ and the frequencies $\left(\alpha_{1}, \ldots, \alpha_{k}\right)$ are the same as for the function $g_{0}(x, t)$.

Proof. Consider the external force $g_{\bar{\omega}}(x, t)=\varphi(x, \bar{\alpha} t+\bar{\omega})$, where $\bar{\omega} \in \mathbb{T}^{k}$. It is clear that $g_{\bar{\omega}} \in \mathcal{H}\left(g_{0}\right)$ (see (14)). By (30) to each such external force $g_{\bar{\omega}}$ there corresponds the unique bounded complete trajectory $z_{\bar{\omega}}(x, t)$ of the Navier-Stokes equation with external force $g_{\bar{\omega}}(x, t)$ that satisfies $(33)$. We set

$$
\Phi(x, \bar{\omega})=z_{\bar{\omega}}(x, 0)
$$

and prove that $\Phi$ is the desired function. First of all we note that

$$
z_{\bar{\omega}}(x, t+h)=z_{\bar{\alpha} h+\bar{\omega}}(x, t) .
$$

This follows from the uniqueness of the bounded complete trajectory $z_{\bar{\alpha} h+\bar{\omega}}(x, t)$ corresponding to the function $g_{\bar{\alpha} h+\bar{\omega}}(x, t)$ and it is easy to see that the function $z_{\bar{\omega}}(x, t+h)$ satisfies the Navier-Stokes system with external force $\varphi(x, \bar{\alpha}(t+h)+\bar{\omega})=g_{\bar{\alpha} h+\bar{\omega}}(x, t)$. By (45) we conclude that

$$
z_{\bar{\omega}}(x, h)=\Phi(x, \bar{\alpha} h+\bar{\omega}),
$$

that is, $z_{\bar{\omega}}(x, t)=\Phi(x, \bar{\alpha} t+\bar{\omega})$ for all $t \in \mathbb{R}$.

We now demonstrate that $\Phi(x, \bar{\omega})=\Phi\left(x, \omega_{1}, \ldots, \omega_{k}\right)$ has the period $2 \pi$ with respect to each argument $\omega_{i}$. This property follows from the uniqueness of bounded complete trajectories because

$$
\Phi\left(x, \bar{\omega}+2 \pi \bar{e}_{i}\right)=z_{\bar{\omega}+2 \pi \bar{e}_{i}}(x, 0)=z_{\bar{\omega}}(x, 0)=\Phi(x, \bar{\omega}) .
$$

Here $\left\{\bar{e}_{i}, i=1, \ldots, k\right\}$ is the standard basis in $\mathbb{R}^{k}$. It only remains to verify the Lipschitz condition with respect to $\bar{\omega} \in \mathbb{T}^{k}$ for the function $\Phi$. We set $w(t)=z_{\bar{\omega}_{1}}(t)-z_{\bar{\omega}_{2}}(t)$. Similarly to (42) we prove the inequality

$$
|w(t)|^{2} \leq|w(\tau)|^{2} C_{2} \mathrm{e}^{-\beta(t-\tau) / 2}+\frac{2}{\beta} C_{2} \int_{\tau}^{t}\left|g_{\bar{\omega}_{1}}(\theta)-g_{\bar{\omega}_{2}}(\theta)\right|^{2} \mathrm{e}^{-\beta(t-\theta) / 2} \mathrm{~d} \theta .
$$

The function $\varphi$ satisfies the inequality

$$
\left|\varphi\left(\bar{\omega}_{1}\right)-\varphi\left(\bar{\omega}_{2}\right)\right| \leq \varkappa\left|\bar{\omega}_{1}-\bar{\omega}_{2}\right| \forall \bar{\omega}_{1}, \bar{\omega}_{2} \in \mathbb{T}^{k} .
$$

Therefore

$$
\left|g_{\bar{\omega}_{1}}(\theta)-g_{\bar{\omega}_{2}}(\theta)\right| \leq \varkappa\left|\bar{\omega}_{1}-\bar{\omega}_{2}\right| .
$$


Hence, from (47) and (48) similarly to (43) and (44) we obtain that

$$
|w(t)|=\left|z_{\bar{\omega}_{1}}(t)-z_{\bar{\omega}_{2}}(t)\right| \leq \varkappa \frac{2 \sqrt{C_{2}}}{\beta}\left|\bar{\omega}_{1}-\bar{\omega}_{2}\right|,
$$

and finally by (45)

$$
\left|\Phi\left(\cdot, \bar{\omega}_{1}\right)-\Phi\left(\cdot, \bar{\omega}_{2}\right)\right|=\left|z_{\bar{\omega}_{1}}(0)-z_{\bar{\omega}_{2}}(0)\right| \leq \varkappa \frac{2 \sqrt{C_{2}}}{\beta}\left|\bar{\omega}_{1}-\bar{\omega}_{2}\right|,
$$

that is, $\Phi(x, \bar{\omega}) \in C^{\mathrm{Lip}}\left(\mathbb{T}^{k} ; H\right)$.

Corollary 2.3. Under the assumptions of Theorem 2.1 the global attractor $\mathcal{A}$ of the Navier-Stokes equation is a Lipschitz-continuous image of the $k$-dimensional torus:

$$
\mathcal{A}=\Phi\left(\mathbb{T}^{k}\right)
$$

and the set $\mathcal{A}$ attracts solutions of the equation with exponential rate (see (33)). Recall that $\Phi(\cdot, \bar{\omega})$ $=\left.\Phi(\cdot, \bar{\alpha} t+\bar{\omega})\right|_{t=0}=\left.z_{\bar{\omega}}(x, t)\right|_{t=0}, \bar{\omega} \in \mathbb{T}^{k}$.

\section{2D NAVIER-Stokes System With RAPIDly OSCILlAting (IN $t$ ) EXTERNAL FORCE}

We consider the 2D Navier-Stokes system of the form

$$
\partial_{t} u+\nu L u+B(u, u)=g(\cdot, t, t / \varepsilon):=g^{\varepsilon},\left.u\right|_{\partial \Omega}=0
$$

$0<\varepsilon \leq \varepsilon_{0}$. We assume that the function $\left.g(x, t, z)\right|_{z=t / \varepsilon}$ is well-defined. For example, $g(x, t, z)$ is continuous as a function of $(t, z)$ with values in $H$. Another example is: $g(x, t, t / \varepsilon)=\Pi g(x, t) \cdot g_{1}(x, t / \varepsilon)$, where $g(x, t) \in L_{2}(\mathbb{R} ; H)$ and $g_{1}(x, t / \varepsilon)$ is a scalar function, $g_{1}(x, z) \in C_{b}\left(\mathbb{R}_{z} ; C(\bar{\Omega})\right)$, $\Pi$ is the orthogonal projector onto $H$.

We suppose that

(i) $g(x, t, t / \varepsilon)$ is translation compact in $L_{2}^{\text {loc }}(\mathbb{R} ; H)$. Then it is known that the following integral is finite:

$$
\|g\|_{L_{2}^{b}}^{2}:=\sup _{t \in \mathbb{R}} \int_{t}^{t+1}|g(\cdot, \tau, \tau / \varepsilon)|_{H}^{2} \mathrm{~d} \tau \leq M^{2}<+\infty
$$

We assume that $M^{2}$ is independent of $\varepsilon$.

(ii) The function $g(x, t, t / \varepsilon)$ has the uniform average $\bar{g}(x, t)$ as $\varepsilon \rightarrow 0+$, that is,

$$
\int_{-T}^{T}\left\langle g\left(\cdot, t^{\prime}+h, \frac{t^{\prime}+h}{\varepsilon}\right), \varphi\left(\cdot, t^{\prime}\right)\right\rangle \mathrm{d} t^{\prime} \rightarrow \int_{-T}^{T}\left\langle\bar{g}\left(\cdot, t^{\prime}+h\right), \varphi\left(\cdot, t^{\prime}\right)\right\rangle \mathrm{d} t^{\prime}
$$

for every function $\varphi(x, t) \in L_{2}(]-T, T\left[, L_{2}(\Omega)\right)$ and every $T>0$ uniformly w.r.t. $h \in \mathbb{R}$. (see [3]). We assume that the function $\bar{g}(x, t)$ is translation compact in $L_{2}^{\operatorname{loc}}(\mathbb{R} ; H)$ as well.

It follows from (i) that equation (51) generates the process $\left\{U_{g^{\varepsilon}}(t, \tau), t \geq \tau, \tau \in \mathbb{R}\right\}, U_{g^{\varepsilon}}(t, \tau) u_{\tau}=u(t)$, where $u(t)$ is the solution of (51). The process $\left\{U_{g^{\varepsilon}}(t, \tau)\right\}$ has an absorbing set $B_{1}$ that is bounded in $V$ and $B_{1}$ is independent of $\varepsilon$ (see (52) and (27)).

Consider some examples of function $g(x, t, t / \varepsilon)$ that satisfy the above conditions (i) and (ii).

1. The function $g(x, t, z)$ is quasiperiodic in $t$ and $z$ with values in $H$, that is,

$$
g(x, t, t / \varepsilon)=\left.G(x, \bar{\eta}, \bar{\omega})\right|_{\bar{\eta}=\bar{\beta} t, \bar{\omega}=\bar{\alpha} t / \varepsilon},
$$


where $\bar{\eta}=\left(\eta_{1}, \ldots, \eta_{m}\right) \in \mathbb{T}^{m}, \bar{\beta}=\left(\beta_{1}, \ldots, \beta_{m}\right) \in \mathbb{R}^{m}, \bar{\omega}=\left(\omega_{1}, \ldots, \omega_{l}\right) \in \mathbb{T}^{l}, \bar{\alpha}=\left(\alpha_{1}, \ldots, \alpha_{l}\right) \in \mathbb{R}^{l}$, and $G(\cdot, \bar{\eta}, \bar{\omega}) \in C^{\operatorname{Lip}}\left(\mathbb{T}^{m} \times \mathbb{T}^{l} ; H\right)$

2. $g(x, t, t / \varepsilon)=\Pi g(x, t) \cdot g_{1}(x, t / \varepsilon)$, where the function $g(x, t)$ is translation compact in the space $L_{2}^{\text {loc }}(\mathbb{R} ; H)$ and the scalar function $g_{1}(x, z)$ is translation compact in $C^{\text {loc }}\left(\mathbb{R}_{z} ; C(\bar{\Omega})\right)$. Besides we assume that the function $g_{1}(x, t / \varepsilon)$ has an average $\bar{g}_{1}(x)$ in $L_{\infty, w *}^{\text {loc }}\left(\mathbb{R} ; L_{\infty}(\Omega)\right)$. Recall that the latter means that

$$
\int_{-T}^{T}\left\langle g_{1}\left(\cdot, \frac{t^{\prime}+h}{\varepsilon}\right), \varphi\left(\cdot, t^{\prime}\right)\right\rangle \mathrm{d} t^{\prime} \rightarrow \int_{-T}^{T}\left\langle\bar{g}_{1}(\cdot), \varphi\left(\cdot, t^{\prime}\right)\right\rangle \mathrm{d} t^{\prime}
$$

for every function $\varphi(x, t) \in L_{1}(]-T, T\left[, L_{1}(\Omega)\right)$ and every $T>0$ uniformly w.r.t. $h \in \mathbb{R}$.

3. Similarly we can set $g(x, t, t / \varepsilon)=\Pi g(x, t) \cdot g_{1}(x, t / \varepsilon)$, where $g(x, t)$ is now a scalar function and $g_{1}(x, z)$ is a vector function. Besides all the results of this section is valid for the functions $g(x, t, t / \varepsilon)$ of the form

$$
\Pi \sum_{i=1}^{N} g_{i}(x, t) g_{1 i}(x, t / \varepsilon),
$$

where the functions $g_{i}(x, t)$ and $g_{1 i}(x, t / \varepsilon)$ belong to the corresponding function spaces described above. Along with equation (51) with external force $g(x, t, t / \varepsilon)$ we consider the averaged equation

$$
\partial_{t} u+\nu L u+B(u, u)=\bar{g}(\cdot, t),\left.u\right|_{\partial \Omega}=0,
$$

where the function $\bar{g}(\cdot, t)$ is the average of the function $g^{\varepsilon}=g(x, t, t / \varepsilon)$ in $L_{2}^{\text {loc }}(\mathbb{R} ; H)$ as $\varepsilon \rightarrow 0+$.

We have proved in Section 2 that equations (51) and (53) have global attractors $\mathcal{A}_{\varepsilon}$ and $\mathcal{A}_{0}$, respectively. Similarly to [9] we prove that $\mathcal{A}_{\varepsilon}$ converges to $\mathcal{A}_{0}$ as $\varepsilon \rightarrow 0+$ in the following sense:

$$
\operatorname{dist}_{H^{1-\delta}}\left(\mathcal{A}_{\varepsilon}, \mathcal{A}_{0}\right) \rightarrow 0 \text { as } \varepsilon \rightarrow 0+,(0<\delta \leq 1) .
$$

In [9] it is proved that $\mathcal{A}_{\varepsilon}$ is also the global attractor of the equation of the form (51) with the external force $\hat{g}^{\varepsilon}(\cdot, t)$, where $\hat{g}^{\varepsilon}(\cdot, t)$ is any function from the hull $\mathcal{H}\left(g^{\varepsilon}\right)$ in $L_{2}^{\text {loc }}(\mathbb{R} ; H)$. We assume that any function $\hat{g}^{\varepsilon}(\cdot, t) \in \mathcal{H}\left(g^{\varepsilon}\right)$ has the form $\hat{g}^{\varepsilon}(\cdot, t)=\hat{g}(\cdot, t, t / \varepsilon)$. We note that this assumption is valid in the all examples above. It can be proved that the average $\hat{g}(\cdot, t)$ of the function $\hat{g}(\cdot, t, t / \varepsilon)$ as $\varepsilon \rightarrow 0+$ belongs to $\mathcal{H}(\bar{g})$.

We need some known estimates for a solution $u(x, t)$ of system (51) (or for the averaged system (53)) with external force $\hat{g}^{\varepsilon}:=\hat{g}\left(\cdot, t, t / \varepsilon\right.$ ) (or with $\hat{g}^{0}:=\hat{g}(\cdot, t)$ ). The proofs of these estimates can be found in $[3,4,6,7,10]$.

We consider the following initial conditions for every $t=\tau, \tau \in \mathbb{R}$ :

$$
\left.u\right|_{t=\tau}=u_{\tau}(x) \text { such that }\left\|u_{\tau}\right\|:=\left\|u_{\tau}\right\|_{V} \leq R,
$$

where $R$ is a fixed (and sufficiently large) real number. The solution $u(x, t)=U_{g}(t, \tau) u_{\tau}$ of the Cauchy problem $(51,55)$, where $g=\hat{g}^{\varepsilon}$ (or of the problem $(53,55)$, where $\bar{g}$ is replaced by $\hat{g}^{0}$ ), satisfies the following inequalities:

$$
\begin{aligned}
|u(\cdot, t)| & =\|u(\cdot, t)\|_{H} \leq R_{0}, R_{0}=R_{0}(R, M) \\
\|u(\cdot, t)\| & =\|u(\cdot, t)\|_{V} \leq R_{1}, R_{1}=R_{1}(R, M) \forall t \geq \tau, \tau \in \mathbb{R} .
\end{aligned}
$$

Moreover,

$$
\begin{aligned}
& \int_{0}^{T}\left\|u\left(\cdot, \tau+t^{\prime}\right)\right\|_{2}^{2} \mathrm{~d} t^{\prime} \leq C(R, M)(T+1) \\
& \int_{0}^{T}\left|\partial_{t} u\left(\cdot, \tau+t^{\prime}\right)\right|^{2} \mathrm{~d} t^{\prime} \leq C(R, M)(T+1) \forall T>0 .
\end{aligned}
$$


We note that $R_{0}(R, M)$ and $R_{1}(R, M)$ in $(56)$ and $C(R, M)$ in $(57,58)$ are independent of $\varepsilon$ and $T$.

The global attractors $\mathcal{A}_{\varepsilon}$ and $\mathcal{A}_{0}$ are uniformly bounded in $V$ :

$$
\left\|\mathcal{A}_{\varepsilon}\right\|_{V} \leq R_{2}, 0 \leq \varepsilon \leq \varepsilon_{0}
$$

where $R_{2}$ is independent of $\varepsilon$ (see [10]). We assume that $R>R_{2}$ (see (55)).

We now formulate some additional assumptions for the difference

$$
\hat{g}^{\varepsilon}(x, t, z)-\hat{g}^{0}(x, t)=\tilde{g}(x, t, z),
$$

where $\hat{g}^{\varepsilon} \in \mathcal{H}\left(g^{\varepsilon}\right), \hat{g}^{0} \in \mathcal{H}\left(g^{0}\right)$ and $\hat{g}^{0}$ is the average of $\hat{g}^{\varepsilon}$ as $\varepsilon \rightarrow 0+$. We denote by $J(x, t, z)$ the primitive with respect to $z$ of the function $\tilde{g}(x, t, z)$ :

$$
\frac{\partial J(x, t, z)}{\partial z}=\tilde{g}(x, t, z), J=\left(J^{1}, J^{2}\right) .
$$

We assume that

$$
\|J(\cdot, t, z)\|_{H} \leq M_{1},\left\|\partial_{t} J(\cdot, t, z)\right\|_{H_{-1}} \leq M_{2} \forall(t, z) \in \mathbb{R} \times \mathbb{R}
$$

where $M_{1}$ and $M_{2}$ are independent of $\hat{g}^{\varepsilon}$.

Lemma 3.1. Assume that the conditions (i, ii), and $(60,61)$ are satisfied for $\hat{g}^{\varepsilon}$ and $\hat{g}^{0}$. Let $u^{\varepsilon}(x, t)$ be a solution of system (51) with external force $\hat{g}^{\varepsilon} \in \mathcal{H}\left(g^{\varepsilon}\right)$ that satisfies the initial condition

$$
\left.u^{\varepsilon}\right|_{t=\tau}=u_{\tau}(x),\left\|u_{\tau}\right\|_{V} \leq R .
$$

Let also $u^{0}(x, t)$ be a solution of the averaged system (53) with external force $\hat{g}^{0} \in \mathcal{H}\left(g^{0}\right)$ and with the same initial condition

$$
\left.u^{\varepsilon}\right|_{t=\tau}=\left.u^{0}\right|_{t=\tau}=u_{\tau}(x)
$$

(Here $\hat{g}^{0}$ is the average of $\hat{g}^{\varepsilon}$ as $\varepsilon \rightarrow 0+$.) Then the difference $v(x, t)=u^{\varepsilon}(x, t)-u^{0}(x, t)$ satisfies the inequality:

$$
|v(\cdot, \tau+t)|=\left|u^{\varepsilon}(\cdot, \tau+t)-u^{0}(\cdot, \tau+t)\right| \leq C \varepsilon^{1 / 2} \mathrm{e}^{\rho t},
$$

where the numbers $C$ and $\rho$ are independent of $\varepsilon, 0<\varepsilon \leq \varepsilon_{0}, \tau \in \mathbb{R}$, and $\hat{g}^{\varepsilon}$.

Proof. The function $v(x, t)$ satisfies the equation

$$
\partial_{t} v+\nu L v+B(v, v)+B\left(v, u^{0}\right)+B\left(u^{0}, v\right)=\hat{g}^{\varepsilon}-\hat{g}^{0}:=\tilde{g} .
$$

Taking the scalar product with $v$ in $H$ we have

$$
\frac{1}{2} \frac{\mathrm{d}}{\mathrm{d} t}|v|^{2}+\nu\|v\|^{2}+(B(v, v), v)+\left(B\left(v, u^{0}\right), v\right)+\left(B\left(u^{0}, v\right), v\right)=\frac{1}{2} \frac{\mathrm{d}}{\mathrm{d} t}|v|^{2}+\nu\|v\|^{2}+\left(B\left(v, u^{0}\right), v\right)=\langle\tilde{g}, v\rangle \cdot
$$

Using the estimate

$$
\left|\left\langle B\left(v, u^{0}\right), v\right\rangle\right| \leq c_{0}|v|\|v\|\|\| u^{0}\left\|\leq \frac{\nu}{2}\right\| v\left\|^{2}+C_{1}|v|^{2}\right\| u^{0} \|^{2}
$$


(see (31)) and integrating (65) in $t$ we obtain that

$$
|v(\tau+t)|^{2} \leq 2 C_{1} \int_{0}^{t}\left\|u^{0}\left(\tau+t^{\prime}\right)\right\|^{2}\left|v\left(\tau+t^{\prime}\right)\right|^{2} \mathrm{~d} t^{\prime}+2 \int_{0}^{t}\left\langle\frac{\partial J\left(\cdot, \tau+t^{\prime},\left(\tau+t^{\prime}\right) / \varepsilon\right)}{\partial z}, v\left(\tau+t^{\prime}\right)\right\rangle \mathrm{d} t^{\prime} .
$$

Here we have used $(60)$ and the fact that $v(\tau)=0$. The last integral in (67) is equal to

$$
\begin{aligned}
& 2 \int_{0}^{t}\left\langle\frac{\partial J\left(\cdot, \tau+t^{\prime},\left(\tau+t^{\prime}\right) / \varepsilon\right)}{\partial z}, v\right\rangle \mathrm{d} t^{\prime}=2\left[\varepsilon \int_{0}^{t}\left\langle\frac{\mathrm{d}}{\mathrm{d} t^{\prime}} J\left(\cdot, \tau+t^{\prime},\left(\tau+t^{\prime}\right) / \varepsilon\right), v\right\rangle \mathrm{d} t^{\prime}\right. \\
& \left.-\varepsilon \int_{0}^{t}\left\langle\frac{\partial J\left(\cdot, \tau+t^{\prime},\left(\tau+t^{\prime}\right) / \varepsilon\right)}{\partial t_{1}^{\prime}}, v\right\rangle \mathrm{d} t^{\prime}\right] \\
= & 2\left[-\varepsilon \int_{0}^{t}\left\langle J\left(\cdot, \tau+t^{\prime},\left(\tau+t^{\prime}\right) / \varepsilon\right), \partial_{t} v\right\rangle \mathrm{d} t^{\prime}+\varepsilon\langle J(\cdot, \tau+t,(\tau+t) / \varepsilon), v(\cdot, \tau+t)\rangle\right] \\
& -2 \varepsilon \int_{0}^{t}\left\langle\frac{\partial J\left(\cdot, \tau+t^{\prime},\left(\tau+t^{\prime}\right) / \varepsilon\right)}{\partial t_{1}^{\prime}}, v\right\rangle \mathrm{d} t^{\prime} \\
\leq & \varepsilon \int_{0}^{t}\left(\left|J\left(\cdot, \tau+t^{\prime},\left(\tau+t^{\prime}\right) / \varepsilon\right)\right|^{2}+\left|\partial_{t} v\left(\cdot, \tau+t^{\prime}\right)\right|^{2}\right) \mathrm{d} t^{\prime} \\
& +2 \varepsilon\langle J(\cdot, \tau+t,(\tau+t) / \varepsilon), v(\cdot, \tau+t)\rangle \\
& -2 \varepsilon \int_{0}^{t}\left\langle\frac{\partial J\left(\cdot, \tau+t^{\prime},\left(\tau+t^{\prime}\right) / \varepsilon\right)}{\partial t_{1}^{\prime}}, v\right\rangle \mathrm{d} t^{\prime},
\end{aligned}
$$

where $\partial J / \partial t_{1}^{\prime}$ denotes the partial derivative of $J$ with respect to the second argument.

Assumption (61) and the estimate (58) implies that the right-hand side of (68) is less or equal to

$$
\varepsilon\left(M_{1}^{2} t+C(M, R)(t+1)\right)+2 \varepsilon M_{1} R_{0}+2 \varepsilon M_{2} R_{1} \leq \varepsilon C_{2}\left(R, M, M_{1}, M_{2}, R_{0}, R_{1}\right)(t+1) .
$$

Therefore, we conclude from $(67,68)$, and $(69)$ that

$$
|v(\cdot, \tau+t)|^{2} \leq 2 C_{1} R_{1} \int_{0}^{t}\left|v\left(\cdot, \tau+t^{\prime}\right)\right|^{2} \mathrm{~d} t^{\prime}+\varepsilon C_{2}(t+1) .
$$

We set $z(t)=|v(\cdot, \tau+t)|^{2} /(t+1)$ and $\rho_{0}=2 C_{1} R_{1}$. Then (70) implies that

$$
z(t) \leq \rho_{0} \int_{0}^{t} \frac{\left(t^{\prime}+1\right)\left|v\left(\cdot, \tau+t^{\prime}\right)\right|^{2}}{\left(t^{\prime}+1\right)(t+1)} \mathrm{d} t^{\prime}+\varepsilon C_{2} \leq \rho_{0} \int_{0}^{t} z\left(t^{\prime}\right) \mathrm{d} t^{\prime}+\varepsilon C_{2} .
$$

Applying Gronwall inequality we have

$$
z(t) \leq \varepsilon C_{2} \mathrm{e}^{\rho_{0} t}
$$

Consequently,

$$
\left|u^{\varepsilon}-u^{0}\right|^{2}=|v(\cdot, \tau+t)|^{2} \leq \varepsilon C_{2}(t+1) \mathrm{e}^{\rho_{0} t} \leq \varepsilon C_{2} \mathrm{e}^{2 \rho t}, 2 \rho=\rho_{0}+1 .
$$

Lemma is proved.

We now assume that the averaged external force $\bar{g}(\cdot, t)$ from the averaged equation (53) satisfies the estimate $(30)$, that is,

$$
\bar{G}:=\frac{\left\|\bar{g}_{0}\right\|_{L_{2}^{b}}}{\lambda \nu^{2}}<\frac{1}{c_{0}}
$$


Then it was shown in Section 2 (see (36)) that the global attractor $\mathcal{A}_{0}$ of equation (53) has the form

$$
\mathcal{A}_{0}=\left[\left\{z_{\bar{g}}(t) \mid t \in \mathbb{R}\right\}\right]_{H}=\bigcup_{\hat{g} \in \mathcal{H}(\bar{g})}\left\{z_{\hat{g}}(0)\right\},
$$

where $\bar{g}$ is fixed in the first equality, while, in the second equality, $\hat{g}$ is taken from the hull $\mathcal{H}(\bar{g})$. Moreover, the unique bounded solution $z_{\hat{g}}(t), t \in \mathbb{R}$ attracts all the solutions $u_{\hat{g}}(t)$ with exponential rate, that is,

$$
\left|u_{\hat{g}}(t)-z_{\hat{g}}(t)\right| \leq C_{0}\left|u_{\tau}-z_{\hat{g}}(\tau)\right| \mathrm{e}^{-\beta(t-\tau)} \forall t \geq \tau
$$

for every $\hat{g} \in \mathcal{H}(\bar{g})$. Here the constants $C_{0}$ and $\beta$ are independent of $\hat{g}$ and $\tau$.

Let us formulate the main result of this section.

Theorem 3.1. Under the assumptions of Lemma 3.1 if (71) holds, then the Hausdorff distance from $\mathcal{A}_{\varepsilon}$ to $\mathcal{A}_{0}$ satisfies the estimate

$$
\operatorname{dist}_{H}\left(\mathcal{A}_{\varepsilon}, \mathcal{A}_{0}\right) \leq C \varepsilon^{\frac{\beta}{2(\beta+\rho)}},
$$

where $\beta$ is taken from (73) and $\rho$ is taken from (63).

Proof. Let $u^{\varepsilon}$ be an arbitrary point of the global attractor $\mathcal{A}_{\varepsilon}$ of equation (51). Using (25) we observe that there exist an external force $\hat{g}^{\varepsilon} \in \mathcal{H}\left(g^{\varepsilon}\right)$ and a bounded complete trajectory $u_{\hat{g}^{\varepsilon}}(t), t \in \mathbb{R}$ such that $u_{\hat{g}^{\varepsilon}}(0)=u^{\varepsilon}$ and $u_{\hat{g}^{\varepsilon}}(t) \in \mathcal{K}_{\hat{g}^{\varepsilon}}$. Recall that $u_{\hat{g}^{\varepsilon}}(t), t \in \mathbb{R}$ is a complete solution of the Navier-Stokes system with external force $\hat{g}^{\varepsilon}$.

Let $\hat{g}^{0}$ be the average of the function $\hat{g}^{\varepsilon}$ as $\varepsilon \rightarrow 0+$. Let also $u_{\hat{g}^{0}}(t-T), t \geq 0$ be the solution of (53) with averaged external force $\hat{g}^{0}$ and with initial conditions

$$
\left.u_{\hat{g}^{0}}\right|_{t=-T}=u_{\hat{g}^{\varepsilon}}(-T) .
$$

The number $T>0$ will be specified below.

Using (73) we obtain that

$$
\left|u_{\hat{g}^{0}}(-T+t)-z_{\hat{g}^{0}}(-T+t)\right| \leq C_{0}\left|u_{\hat{g}^{\varepsilon}}(-T)-z_{\hat{g}^{0}}(-T)\right| \mathrm{e}^{-\beta t} \forall t \geq 0,
$$

where $z_{\hat{g}^{0}}(t)$ is the unique bounded (in $H$ ) complete solution of (53) with external force $\hat{g}^{0}$. Recall that the global attractors $\mathcal{A}_{\varepsilon}$ are uniformly (w.r.t. $\left.0 \leq \varepsilon<\varepsilon_{0}\right)$ bounded in $H:\left|\mathcal{A}_{\varepsilon}\right| \leq R_{0}$, therefore $\left|u_{\hat{g}^{\varepsilon}}(-T)\right|,\left|z_{\hat{g}^{0}}(-T)\right| \leq R_{0}$ and (76) implies that

$$
\left|u_{\hat{g}^{0}}(-T+t)-z_{\hat{g}^{0}}(-T+t)\right| \leq C_{1} \mathrm{e}^{-\beta t} \forall t \geq 0
$$

where $C_{1}=C_{0} R_{0}$.

From (63) we conclude that

$$
|v(\cdot,-T+t)|:=\left|u_{\hat{g}^{\varepsilon}}(\cdot,-T+t)-u_{\hat{g}^{0}}(\cdot,-T+t)\right| \leq C \varepsilon^{1 / 2} \mathrm{e}^{\rho t} .
$$

We now set $t=T$ in $(77,78)$ and obtain that

$$
\begin{aligned}
\mid u_{\hat{g}^{\varepsilon}}(\cdot,-T+T) & -z_{\hat{g}^{0}}(\cdot,-T+T)|=| u_{\hat{g}^{\varepsilon}}(\cdot, 0)-z_{\hat{g}^{0}}(\cdot, 0) \mid \\
& \leq\left|u_{\hat{g}^{\varepsilon}}(\cdot, 0)-u_{\hat{g}^{0}}(\cdot, 0)\right|+\left|u_{\hat{g}^{0}}(\cdot, 0)-z_{\hat{g}^{0}}(\cdot, 0)\right| \\
& \leq C_{0} \varepsilon^{1 / 2} \mathrm{e}^{\rho T}+C_{1} \mathrm{e}^{-\beta T} .
\end{aligned}
$$


We now choose $T$ such that

$$
\varepsilon^{1 / 2} \mathrm{e}^{\rho T}=\mathrm{e}^{-\beta T}
$$

that is,

$$
T=\frac{1}{2(\rho+\beta)} \log \left(\frac{1}{\varepsilon}\right)
$$

Therefore from (79) we finally have

$$
\left|u_{\hat{g}^{\varepsilon}}(\cdot, 0)-z_{\hat{g}^{0}}(\cdot, 0)\right| \leq 2 C_{2} \varepsilon^{\frac{\beta}{2(\rho+\beta)}} .
$$

Since $u^{\varepsilon}=u_{\hat{g}^{\varepsilon}}(\cdot, 0)$ was an arbitrary point of $\mathcal{A}_{\varepsilon}$ and $z_{\hat{g}^{0}}(\cdot, 0) \in \mathcal{A}_{0}$ we conclude from (81) that

$$
\operatorname{dist}_{H}\left(\mathcal{A}_{\varepsilon}, \mathcal{A}_{0}\right) \leq C \varepsilon^{\frac{\beta}{2(\beta+\rho)}} .
$$

We now consider some examples of functions $g(x, t, z)$ that satisfy $(60)$ and $(61)$.

a) Let $g(x, t, z)$ be trigonometrical polynomial with respect to $z$ with frequencies $\left(\alpha_{1}, \ldots, a_{l}\right)=\bar{\alpha}$ which are rationally independent:

$$
g(x, t, z)=a_{0}(x, t)+\sum_{0<|\bar{k}| \leq N} a_{\bar{k}}(x, t) \mathrm{e}^{i(\bar{\alpha}, \bar{k}) z}:=g^{0}(x, t)+\tilde{g}(x, t, z) .
$$

Here $\bar{k}=\left(k_{1}, k_{2}, \ldots, k_{l}\right) \in \mathbb{Z}^{l}$ and $|\bar{k}|=k_{1}+\ldots+k_{l}$. We assume that $\overline{a_{\bar{k}}}=a_{-\bar{k}}$ for $0<|\bar{k}| \leq N$ and the functions $a_{0}(x, t)$ and $a_{\bar{k}}(x, t)$ are translation compact in $C^{\text {loc }}(\mathbb{R} ; H)$. In particular they are bounded in $C_{b}(\mathbb{R} ; H)$. Then clearly $g^{0}(x, t)=a_{0}(x, t)$ is the average of $g(x, t, t / \varepsilon)$ as $\varepsilon \rightarrow 0+$. In this case

$$
J(x, t, z)=\sum_{0<|\bar{k}| \leq N} \frac{a_{\bar{k}}(x, t)}{i(\bar{\alpha}, \bar{k})} \mathrm{e}^{i(\bar{\alpha}, \bar{k}) z}, \frac{\partial J(x, t, z)}{\partial z}=\tilde{g}(x, t, z) .
$$

Since the numbers $\alpha_{j}$ are rationally independent we have

$$
|(\bar{\alpha}, \bar{k})| \geq \delta>0 \quad \forall \bar{k}: 0<|\bar{k}| \leq N
$$

The function $J(x, t, z)$ satisfies the first inequality in (61) because

$$
\|J(\cdot, t, z)\|_{H} \leq \sum_{0<|\bar{k}| \leq N} \frac{\left\|a_{\bar{k}}(\cdot, t)\right\|_{H}}{\delta} \leq M_{1} \forall(t, z) \in \mathbb{R} \times \mathbb{R} .
$$

Besides we assume that $\partial_{t} a_{\bar{k}}(x, t) \in C_{b}\left(\mathbb{R} ; H_{-1}\right)$ and $\partial_{t} a_{\bar{k}}(x, t)$ are translation compact in this space for all $\bar{k}$ such that $0<|\bar{k}| \leq N$. Then

$$
\sum_{0<|\bar{k}| \leq N} \sup _{t \in \mathbb{R}}\left\|\partial_{t} a_{\bar{k}}(\cdot, t)\right\|_{H_{-1}}<+\infty
$$

because each summand in (85) is bounded. Hence,

$$
\left\|\partial_{t} J(\cdot, t, z)\right\|_{H_{-1}} \leq M_{2}<+\infty \forall(t, z) \in \mathbb{R} \times \mathbb{R}
$$

It follows from (82-84), and (86) that any function $\hat{g}(x, t, t / \varepsilon)$ from the hull $\mathcal{H}(g)$ has the form $(82)$ and satisfies conditions (84) and (86) which implies condition (61) for $\hat{g}(x, t, t / \varepsilon)$ as well. 
b) We now consider the case, where the external force $g(x, t, z)(z=t / \varepsilon)$ is quasiperiodic with respect to $z$, that is,

$$
g(x, t, z)=G(x, t, z \bar{\alpha}), \bar{\alpha}=\left(\alpha_{1}, \ldots, \alpha_{l}\right)
$$

where $G(x, t, \bar{\omega})=G\left(x, t, \omega_{1}, \ldots, \omega_{l}\right)$ is $2 \pi$-periodic function with respect to each $\omega_{i}(i=1, \ldots, l)$. We set

$$
g^{0}(x, t)=\frac{1}{(2 \pi)^{l}} \int_{\mathbb{T}^{l}} \tilde{G}(x, t, \bar{\omega}) \mu(\mathrm{d} \bar{\omega}) \text { and } \tilde{G}(x, t, \bar{\omega})=G(x, t, \bar{\omega})-g^{0}(x, t) .
$$

Then

$$
g(x, t, z)=g^{0}(x, t)+\tilde{g}(x, t, z),
$$

where $\tilde{g}(x, t, z)=\tilde{G}(x, t, z \bar{\alpha})$. It is obvious that

$$
\frac{1}{(2 \pi)^{l}} \int_{\mathbb{T}^{l}} \tilde{G}(x, t, \bar{\omega}) \mu(\mathrm{d} \bar{\omega})=0 \forall x \in \bar{\Omega}, t \in \mathbb{R} .
$$

Here $\mu(d \bar{\omega})$ is the Lebesgue measure in $\mathbb{T}^{l}$ and $\mu\left(\mathbb{T}^{l}\right)=(2 \pi)^{l}$. For the simplicity we assume that $\tilde{G}(x, t, \bar{\omega})$ $\in C\left(\mathbb{R} \times \mathbb{T}^{l} ; H\right)$. Besides we assume that the numbers $\bar{\alpha}=\left(\alpha_{1}, \ldots, \alpha_{l}\right)$ are rationally independent and satisfy the following Diophantine condition:

$$
|(\bar{\alpha}, \bar{k})| \geq C_{\bar{\alpha}}|\bar{k}|^{-(l-1+\delta)} \quad \forall \bar{k} \in \mathbb{Z}^{l} \backslash\{0\}(\delta>0) .
$$

It is known that (88) holds if $\bar{\alpha}$ belongs to a set $\mathbb{R}^{l} \backslash Q:=S^{l}$, where the set $Q$ has zero Lebesgue measure in $\mathbb{R}^{l}: \mu(Q)=0$ (see $\left.[11,12]\right)$. Let $\bar{\alpha} \in S^{l}$. We expand $\tilde{G}(x, t, \bar{\omega})$ into a Fourier-series with respect to $\omega_{i}$ $(i=1, \ldots, l)$ and set $\omega_{i}=z \alpha_{i}, z \in \mathbb{R}$. We obtain the formula

$$
\tilde{g}(x, t, z)=\tilde{G}(x, t, z \bar{\alpha})=\sum_{\bar{k} \neq 0} a_{\bar{k}}(x, t) \mathrm{e}^{i(\bar{\alpha}, \bar{k}) z} .
$$

Similarly to (83) we set

$$
J(x, t, z)=\sum_{\bar{k} \in \mathbb{Z}^{l} \backslash\{0\}} \frac{a_{\bar{k}}(x, t)}{i(\bar{\alpha}, \bar{k})} \mathrm{e}^{i(\bar{\alpha}, \bar{k}) z} .
$$

As above we assume that the functions $a_{\bar{k}}(x, t)$ are translation compact in $C^{\text {loc }}(\mathbb{R} ; H)$ and $\partial_{t} a_{\bar{k}}(x, t)$ are translation compact in $C^{\text {loc }}\left(\mathbb{R} ; H_{-1}\right)$ for $\bar{k} \in \mathbb{Z}^{l} \backslash\{0\}$. If the series

$$
\sum_{\bar{k} \neq 0} \sup _{t \in \mathbb{R}}\left|a_{\bar{k}}(\cdot, t)\right||\bar{k}|^{l-1+\delta}<+\infty,
$$

then clearly

$$
\|J(\cdot, t, z)\|_{H} \leq M_{1} \forall(t, z) \in \mathbb{R} \times \mathbb{R} .
$$

We note that estimates (91) and (92) hold for every $\tilde{g}(x, t, t / \varepsilon)=\hat{g}(x, t, t / \varepsilon)-\hat{g}^{0}(x, t)$, where $\hat{g}(x, t, t / \varepsilon) \in \mathcal{H}(g)$ and $\hat{g}^{0}(x, t)$ is the average of $\hat{g}(x, t, t / \varepsilon)$ as $\varepsilon \rightarrow 0+$. Therefore the first assumption from (61) is verified for 
$J(x, t, z)$. Similarly to example a) we formulate the sufficient conditions for $\partial_{t} \tilde{g}(x, t, z)$ that guarantees the second assumption from (61), that is,

$$
\left\|\partial_{t} J(\cdot, t, z)\right\|_{H_{-1}} \leq M_{2} \forall(t, z) \in \mathbb{R} \times \mathbb{R} .
$$

\section{2D NAVIER-Stokes System With QUASIPERIOdic (IN $t$ AND $z=t / \varepsilon$ ) EXTERNAL FORCE}

We consider system (51) with external force of the form $g(x, t, t / \varepsilon)$, where $g(x, t, z)$ is a quasiperiodic function with respect to $t$ and $z$, that is,

$$
g(x, t, t / \varepsilon)=G(x, t \bar{\gamma}, z \bar{\alpha}), \bar{\gamma}=\left(\gamma_{1}, \ldots, \gamma_{m}\right), \bar{\alpha}=\left(\alpha_{1}, \ldots, \alpha_{l}\right) .
$$

Here $G(x, \bar{\eta}, \bar{\omega})=G\left(x, \eta_{1}, \ldots, \eta_{m}, \omega_{1}, \ldots, \omega_{l}\right)$ is $2 \pi$-periodic function with respect to each $\eta_{i}(i=1, \ldots, m)$ and each $\omega_{i}(i=1, \ldots, l)$. Moreover we assume that $G(x, \bar{\eta}, \bar{\omega}) \in C^{\operatorname{Lip}}\left(\mathbb{T}^{m+l} ; H\right)$ and the real numbers $(\bar{\gamma}, \bar{\alpha})$ $=\left(\gamma_{1}, \ldots, \gamma_{m}, \alpha_{1}, \ldots, \alpha_{l}\right)$ are rationally independent. Besides let the numbers $\left(\alpha_{1}, \ldots, \alpha_{l}\right)$ satisfy the Diophantine condition (88) and let the assumptions given in the above example b) be valid that provide (91) and (93).

Let also the Grashof number for equation (51) with external force $g(x, t, t / \varepsilon)$ satisfies inequality (30), that is,

$$
G^{\varepsilon}:=\frac{\left\|g^{\varepsilon}\right\|_{L_{2}^{b}}}{\lambda \nu^{2}}<\frac{1}{c_{0}}, 0<\varepsilon \leq \varepsilon_{0}
$$

Then it was shown in Section 2 (see (50)) that the global attractor of this equation

$$
\mathcal{A}_{\varepsilon}=Q^{\varepsilon}\left(\mathbb{T}^{m+l}\right), \quad 0<\varepsilon \leq \varepsilon_{0}
$$

is a Lipschitz-continuous image of a $(m+l)$-dimensional torus $\mathbb{T}^{m+l}$. We also consider the corresponding averaged equation with external force $g^{0}(x, t):=\bar{g}(x, t)$, where $\bar{g}(x, t)$ is the average of $g(x, t, t / \varepsilon)$ as $\varepsilon \rightarrow 0+$. It is known that

$$
\bar{g}(x, t)=\frac{1}{(2 \pi)^{l}} \int_{\mathbb{T}^{l}} g(x, t, z) \mu(\mathrm{d} z) .
$$

From (94) we conclude that the corresponding Grashof number $G^{0}$ satisfies the following inequality:

$$
G^{0}:=\frac{\left\|g^{0}\right\|_{L_{2}^{b}}}{\lambda \nu^{2}}<\frac{1}{c_{0}} .
$$

Then by Corollary 2.3 the global attractor $\mathcal{A}_{0}$ is a Lipschitz-continuous image of the $m$-dimensional torus $\mathbb{T}^{m}$ :

$$
\mathcal{A}_{0}=Q^{0}\left(\mathbb{T}^{m}\right) .
$$

Since all the assumptions of Lemma 3.1 and Theorem 3.1 are satisfied, then by (74)

$$
\operatorname{dist}_{H}\left(\mathcal{A}_{\varepsilon}, \mathcal{A}_{0}\right)=\operatorname{dist}_{H}\left(Q^{\varepsilon}\left(\mathbb{T}^{m+l}\right), Q^{0}\left(\mathbb{T}^{m}\right)\right) \leq C \varepsilon^{\frac{\beta}{2(\beta+\rho)}} .
$$

Here, as in Section 3, $\beta$ is the coefficient of the exponential attraction of bounded sets to the global attractor $\mathcal{A}_{0}$ of the averaged equation (53) and $\rho$ is the coefficient of the exponential divergency of solutions of equations (51) and (53) with the same initial conditions (see (63)). 


\section{REFERENCES}

[1] A. Haraux, Systèmes dynamiques dissipatifs et applications. Masson, Paris, Milan, Barcelona, Rome (1991).

[2] V.V. Chepyzhov and M.I. Vishik, Attractors of non-autonomous dynamical systems and their dimension. J. Math. Pures Appl. 73 (1994) 279-333.

[3] V.V. Chepyzhov and M.I. Vishik, Attractors for equations of mathematical physics. AMS, Providence, Rhode Island (2002).

[4] J.-L. Lions, Quelques méthodes de résolution des problèmes aux limites non linéaires. Dunod Gauthier-Villars, Paris (1969).

[5] O.A. Ladyzhenskaya, The mathematical theory of viscous incompressible flow. Moscow, Nauka (1970). English transl.: Gordon and Breach, New York (1969).

[6] R. Temam, Infinite-dimensional dynamical systems in mechanics and physics. New York, Springer-Verlag, Appl. Math. Ser. 68 (1988), 2nd Ed. 1997.

[7] A.V. Babin and M.I. Vishik, Attractors of evolution equations. Nauka, Moscow (1989). English transl.: North Holland (1992).

[8] V.V. Chepyzhov and A.A. Ilyin, On the fractal dimension of invariant sets; applications to Navier-Stokes equations (to appear).

[9] M.I. Vishik and V.V. Chepyzhov, Averaging of trajectory attractors of evolution equations with rapidly oscillating terms. Mat. Sbornik 192 (2001) 16-53. English transl.: Sbornik: Mathematics 192 (2001).

[10] V.V. Chepyzhov and M.I. Vishik, Trajectory attractors for 2D Navier-Stokes systems and some generalizations. Topol. Meth. Nonl. Anal., J.Juliusz Schauder Center 8 (1996) 217-243.

[11] J.W.S. Kassels, An introduction to Diophantine approximations. Cambridge University Press (1957).

[12] B. Fiedler and M.I. Vishik, Quantitative homogenization of global attractors for reaction-diffusion systems with rapidly oscillating terms. Preprint (2000). 